\title{
DUAL OPERATIONS ON SADDLE FUNCTIONS
}

\author{
BY \\ L. McLINDEN(1)
}

\begin{abstract}
Dual operations on convex functions play a central role in the analysis of constrained convex optimization problems. Our aim here is to provide tools for a similar analysis of constrained concave-convex minimax problems. Two pairs of dual operations on convex functions, including addition and infimal convolution, are extended to saddle functions. For the resulting saddle functions much detailed information is given, including subdifferential formulas. Also, separable saddle functions are defined and some basic facts about them established.
\end{abstract}

Introduction. Constrained convex optimization problems have been analyzed quite successfully in recent years via the dual approach. Of central importance in this analysis have been pairs of dual operations on convex functions. Addition and infimal convolution are perhaps the most familiar such pair of operations. Another, perhaps equally important dual pair of operations involves combining a convex function with a linear transformation in two different ways. In this paper both of these dual pairs of operations are extended to saddle functions on finite-dimensional spaces, and very detailed information, including subdifferential formulas, is given concerning the saddle functions which result from these operations. The present results will be applied in [4] and subsequent papers to the analysis of constrained saddle point problems.

In working with saddle functions one confronts two major complications not encountered in convex function theory. The first of these arises from the fact that, for applications to saddle point problems, the natural objects of attention are equivalence classes of functions rather than individual functions. This means that in developing operations for saddle functions one is interested really in operations for these equivalence classes of functions. That is, one wants the operations to be independent, up to equivalence, of the particular choice of representatives of the equivalence classes involved. A second complication stems from the fact that saddle functions involve two arguments, one of which is naturally associated with minimization and the other with maximization. This of ten leads to the ambiguity of having to decide between taking the "sup inf" or the "inf sup" of some saddle function.

Received by the editors May 4, 1972.

AMS (MOS) subject classifications (1970). Primary 90C25, 90C30, 90D05; Secondary 26A51, 49A40.

Key words and phrases. Convex analysis, minimax theory, constrained concave-convex problems, dual extremum problems, conjugate saddle functions, equivalence classes, dual operations, addition, extremal convolution, composition with linear transformations, subdifferential formulas, duality formulas, stability of solutions, separable saddle functions.

(1) Sponsored by the United States Army under Contract DA-31-124-ARO-D-462. 
These complications make the extension of results from convex function theory to saddle function theory far from routine. In view of this, it is a surprising but happy fact that much can be extended to saddle function theory, as will be illustrated in this and subsequent papers.

The dual approach to minimax theory began in 1964 with the papers of Moreau [5] and Rockafellar [6]. In fact, it was in [6] that the two complications mentioned above were first recognized and handled successfully. In particular, it was here that the notion of equivalence between saddle functions originated and here also that the concepts of conjugacy and of subdifferential were extended to equivalence classes of saddle functions. With the aid of these fundamental tools, the questions of existence and characterization of the optimal values and optimal solutions of unconstrained concave-convex saddle point problems were answered fairly completely.

Further work concerning the dual approach to saddle point problems can be found in Rockafellar [8], [9], [10], [11], Lebedev-Tynjanskii [2], Tynjanskii [12] and McLinden [3], [4]. The conjugacy correspondence for saddle functions was developed independently in the 1969 paper of Tynjanskiî, although for a narrower class of functions than that treated in [6]. Concerning our results, Gossez [1] independently has essentially defined the addition operation for equivalence classes of closed proper saddle functions on Banach spaces and obtained results for it comparable to those of Theorem 1 below.

The rest of this paper is in two parts. In the first we develop the two dual pairs of operations promised and present our results concerning them together with some discussion. The actual proofs are presented separately in the second part of the paper.

Acknowledgment. The present work took preliminary form in a thesis written under Professor R. Tyrrell Rockafellar at the University of Washington. The author takes this opportunity to express his gratitude to Professor Rockafellar for supplying encouragement and thoughtful comments, as well as for posing the problem of developing dual operations on equivalence classes of saddle functions.

1. Results and discussion. The definitions we use are those in Rockafellar [10] unless otherwise stated, and for convenience we use the following additional notation. Suppose $K$ and $L$ are saddle functions. We write $K \sim L$ if and only if $K$ is equivalent to $L$, and we let $[K]$ denote the equivalence class to which $K$ belongs. Frequently we use $\tilde{K}$ to denote a general element of $[K]$. When $K$ is closed, the unique least and greatest elements of $[K]$ are denoted by $\underline{K}$ and $\bar{K}$, respectively, and the equivalence class conjugate to $[K]$ is denoted by $\left[K^{*}\right]$. Thus when $K$ is closed, its lower and upper conjugates are $\underline{K}^{*}$ and $\bar{K}^{*}$, respectively, while a general element of $\left[K^{*}\right]$ is denoted by $\tilde{K}^{*}$.

Throughout the paper we use the conventions set forth in [10, p. 24] concerning arithmetic calculations involving $+\infty$ and $-\infty$. In particular, sup $\varnothing=-\infty$ and inf $\varnothing=+\infty$. 
Recall that the notions of (lower and upper) saddle value, saddle point, effective domain, proper, closed, and subdifferential, as well as the notion of (lower and upper) conjugate for closed saddle functions, are each invariant under equivalence of saddle functions. Therefore, these are also notions concerning entire equivalence classes, and consequently we often use them as such. For instance, we say that " $[K]$ is a closed, proper equivalence class," or refer to "the subdifferential of $[K]$," etc.

Note that the term "saddle function" can mean either a concave-convex or a convex-concave function. Without loss of generality, in this paper we deal always with concave-convex functions.

Suppose $\left[K_{1}\right], \ldots,\left[K_{r}\right]$ are equivalence classes of concave-convex functions on $R^{m} \times R^{n}$, and write $C=\bigcap_{i=1}^{r} \operatorname{dom}_{1} K_{i}$ and $D=\bigcap_{i=1}^{r} \operatorname{dom}_{2} K_{i}$. We say that the sum of $\left[K_{1}\right], \ldots,\left[K_{r}\right]$ is well-defined if and only if all the concave-convex functions of the form

$$
\begin{aligned}
(x, y) & \rightarrow \tilde{K}_{1}(x, y)+\ldots+\tilde{K}_{r}(x, y) & & \text { if } x \in C, \\
& \rightarrow-\infty & & \text { if } x \notin C,
\end{aligned}
$$

or

$$
\begin{array}{rlrl}
(x, y) & \rightarrow \tilde{K}_{1}(x, y)+\ldots+\tilde{K}_{r}(x, y) & & \text { if } y \in D, \\
& \rightarrow+\infty & \text { if } y \notin D,
\end{array}
$$

for $\tilde{K}_{1} \in\left[K_{1}\right], \ldots, \tilde{K}_{r} \in\left[K_{r}\right]$, belong to a single equivalence class, denoted by $\left[K_{1}+\ldots+K_{r}\right]$. The operation which sends $\left[K_{1}\right], \ldots,\left[K_{r}\right]$ into $\left[K_{1}+\ldots+K_{r}\right]$ is naturally called addition. Note that this definition copes with the technical difficulty of adding together extended-real-valued functions.

When each of $\left[K_{1}\right], \ldots,\left[K_{r}\right]$ is closed, it is not hard to see that the condition in this definition is the same as requiring that the two particular concave-convex functions

$$
\begin{aligned}
(x, y) & \rightarrow \underline{K}_{1}(x, y)+\ldots+\underline{K}_{r}(x, y) & & \text { if } x \in C, \\
& \rightarrow-\infty & & \text { if } x \notin C,
\end{aligned}
$$

and

$$
\begin{aligned}
(x, y) & \rightarrow \bar{K}_{1}(x, y)+\ldots+\bar{K}_{r}(x, y) & & \text { if } y \in D, \\
& \rightarrow+\infty & & \text { if } y \notin D,
\end{aligned}
$$

be equivalent. In [1] Gossez has defined the sum of two saddle functions in this way and proved a close variant of the following theorem in the context of Banach spaces. 
Theorem 1. Let $\left[K_{1}\right], \ldots,\left[K_{r}\right]$ be equivalence classes of closed proper concaveconvex functions on $R^{m} \times R^{n}$, and assume $\bigcap_{i=1}^{r}$ ri $\left(\operatorname{dom} K_{i}\right) \neq \varnothing$. Then

(i) $\left[K_{1}+\ldots+K_{r}\right]$ is well-defined, closed and proper with

$$
\operatorname{dom}\left(K_{1}+\ldots+K_{r}\right)=\operatorname{dom} K_{1} \cap \ldots \cap \operatorname{dom} K_{r}
$$

(ii) the least and greatest elements of $\left[K_{1}+\ldots+K_{r}\right]$ are the functions

$$
\begin{aligned}
(x, y) & \rightarrow \underline{K}_{1}(x, y)+\ldots+\underline{K}_{r}(x, y) & & \text { if } x \in C, \\
& \rightarrow-\infty & & \text { if } x \notin C,
\end{aligned}
$$

and

$$
\begin{array}{rlrl}
(x, y) & \rightarrow \bar{K}_{1}(x, y)+\ldots+\bar{K}_{r}(x, y) & & \text { if } y \in D, \\
& \rightarrow+\infty & \text { if } y \notin D,
\end{array}
$$

respectively, where $C=\bigcap_{i=1}^{r} \operatorname{dom}_{1} K_{i}$ and $D=\bigcap_{i=1}^{r} \operatorname{dom}_{2} K_{i}$; and

(iii) the subdifferential of $\left[K_{1}+\ldots+K_{r}\right]$ is given by

$$
\partial\left(K_{1}+\ldots+K_{r}\right)(x, y)=\partial K_{1}(x, y)+\ldots+\partial K_{r}(x, y) .
$$

Next we extend the operation of infimal convolution to saddle functions. Suppose again that $\left[K_{1}\right], \ldots,\left[K_{r}\right]$ are equivalence classes of concave-convex functions on $R^{m} \times R^{n}$, and write dom $K_{i}=C_{i} \times D_{i}$. We say that the extremal convolute of $\left[K_{1}\right], \ldots,\left[K_{r}\right]$ is well-defined if and only if all the concave-convex functions of the form

$$
(x, y) \rightarrow \sup _{\Sigma x_{i}=x ; x_{i} \in C_{i}} \inf _{\Sigma y_{i}=y} \sum \tilde{K}_{i}\left(x_{i}, y_{i}\right)
$$

or of the form

$$
(x, y) \rightarrow \inf _{\Sigma y_{i}=y ; y_{i} \in D_{i}} \sup _{\sum x_{i}=x} \sum \tilde{K}_{i}\left(x_{i}, y_{i}\right)
$$

for $\tilde{K}_{1} \in\left[K_{1}\right], \ldots, \tilde{K}_{r} \in\left[K_{r}\right]$, belong to a single equivalence class, denoted by $\left[K_{1} \square \ldots \square K_{r}\right]$. The operation which sends $\left[K_{1}\right], \ldots,\left[K_{r}\right]$ into $\left[K_{1} \square \ldots \square K_{r}\right]$ is called extremal convolution. Many things can be said about this seemingly complex operation, as shown by the next two theorems.

Theorem 2. Let $\left[K_{1}\right], \ldots,\left[K_{r}\right]$ be equivalence classes of closed proper concaveconvex functions on $R^{m} \times R^{n}$, and assume $\bigcap_{i=1}^{r} \operatorname{ri}\left(\operatorname{dom} K_{i}^{*}\right) \neq \varnothing$. Then $\left[K_{1} \square\right.$.. $\left.\square K_{r}\right]$ is well-defined, closed and proper with $\operatorname{dom}\left(K_{1} \square \ldots \square K_{r}\right) \subset \operatorname{dom} K_{1}$ $+\ldots+$ dom $K_{r}$. Moreover $\left[\left(K_{1} \square \ldots \square K_{r}\right)^{*}\right]=\left[K_{1}^{*}+\ldots+K_{r}^{*}\right]$.

Theorems 1 and 2 together establish a single, simple condition under which "the conjugate of the sum is the extremal convolute of the conjugates," that is, under which the operations of addition and extremal convolution are the duals 
of each other with respect to the conjugacy correspondence.

The subdifferential of $\left[K_{1} \square \ldots \square K_{r}\right]$ can be given an immediate characterization by means of the conjugacy formula of Theorem 2 and the subdifferential formula of Theorem 1. For this one just needs the fact that, for $K$ closed, $\partial K^{*}$ is just the inverse of $\partial K$ in the sense of multivalued mappings ([10, Theorem 37.5] or [11, Theorem 7]).

From a comparison with the operation of infimal convolution for convex functions, one might expect that the inclusion of Theorem 2 could be strengthened to equality, or at least supplemented by the inclusion

$$
\text { ri(dom } \left.K_{1}+\ldots+\operatorname{dom} K_{r}\right) \subset \operatorname{dom}\left(K_{1} \square \ldots \square K_{r}\right) .
$$

But in general, even this latter relation can fail drastically, as is seen by taking $r=2, m=n, K_{1}(x, y)=\langle x, y\rangle$ and $K_{2}(x, y)=-\langle x, y\rangle$. In this case dom $K_{1}$ $+\operatorname{dom} K_{2}=R^{m} \times R^{n}$ while $\operatorname{dom}\left(K_{1} \square K_{2}\right)=\{0\} \times\{0\}$.

Various criteria can be given, though, which will ensure that one of these two relations does hold. They are essentially growth conditions on the $K_{i}$ or $K_{i}^{*}$. The simplest such condition (but by no means the most general) is that $\left[K_{1}^{*}\right.$ $\left.+\ldots+K_{r}^{*}\right]$ be "cofinite," i.e. closed and proper with a finite conjugate. It can be shown that a closed proper saddle function is cofinite if its effective domain is bounded. Thus, under the hypotheses of Theorem 2,

$$
R^{m} \times R^{n}=\operatorname{dom}\left(K_{1} \square \ldots \square K_{r}\right)=\operatorname{dom} K_{1}+\ldots+\operatorname{dom} K_{r}
$$

if $\bigcap_{i=1}^{r}$ dom $K_{i}^{*}$ is bounded. We shall not pursue this further here, since we shall give a general discussion of growth conditions for saddle functions in another paper.

Except for the possibility just mentioned that $\operatorname{dom}\left(K_{1} \square \ldots \square K_{r}\right)$ may "collapse," Theorem 2 shows that extremal convolution satisfies several of the important properties enjoyed by infimal convolution. The next theorem carries this even further by asserting that the complicated minimax extrema appearing in the definition of $\left[K_{1} \square \ldots \square K_{r}\right]$ are usually attained in a very strong sense.

Theorem 3. Let the $\left[K_{i}\right]$ be as in Theorem 2, write dom $K_{i}=C_{i} \times D_{i}$, and pick any $\tilde{K}_{1} \in\left[K_{1}\right], \ldots, \tilde{K}_{r} \in\left[K_{r}\right]$. Then the least and greatest elements of $\left[K_{1} \square \ldots\right.$ $\left.K_{r}\right]$ are the functions

$$
\underline{H}(x, y)=\sup _{\Sigma x_{i}=x ; x_{i} \in C_{i}}\left\{\operatorname{cl}\left(\tilde{K}_{1}\left(x_{1}, \cdot\right) \square \ldots \square \tilde{K}_{r}\left(x_{r}, \cdot\right)\right)(y)\right\}
$$

and

$$
\bar{H}(x, y)=\inf _{\Sigma y_{i}=y ; y_{i} \in D_{i}}\left\{\operatorname{cl}\left(\tilde{K}_{1}\left(\cdot, y_{1}\right) \square \ldots \square \tilde{K}_{r}\left(\cdot, y_{r}\right)\right)(x)\right\},
$$

respectively, where $\square$ operating on a convex (resp. concave) function denotes infimal (resp. supremal) convolution. Moreover, for each $(x, y) \in \operatorname{dom} \partial\left(K_{1} \square \ldots \square K_{r}\right)$ the 
extrema appearing in the definitions of $\underline{H}(x, y)$ and $\bar{H}(x, y)$ are attained, in the sense that there exist pairs $\left(x_{1}, y_{1}\right) \in \operatorname{dom} \partial K_{1}, \ldots,\left(x_{r}, y_{r}\right) \in \operatorname{dom} \partial K_{r}$, such that $\sum x_{i}$ $=x, \sum y_{i}=y$ and the quantities

$$
\underline{H}(x, y)=\operatorname{cl}\left(\tilde{K}_{1}\left(x_{1}, \cdot\right) \square \ldots \square \tilde{K}_{r}\left(x_{r}, \cdot\right)\right)(y)=\liminf _{y^{\prime} \rightarrow y}\left\{\inf _{\Sigma y_{i}^{\prime}=y^{\prime}} \sum \tilde{K}_{i}\left(x_{i}, y_{i}^{\prime}\right)\right\}
$$

and

$$
\left.\bar{H}(x, y)=\operatorname{cl}\left(\tilde{K}_{1}\left(\cdot, y_{1}\right) \square \ldots \square \tilde{K}_{r}\left(\cdot, y_{r}\right)\right)(x)=\lim _{x^{\prime} \rightarrow x} \sup _{\sum x_{i}^{\prime}=x^{\prime}} \sum \tilde{K}_{i}\left(x_{i}^{\prime}, y_{i}\right)\right\}
$$

are finite and equal to $\tilde{K}_{1}\left(x_{1}, y_{1}\right)+\ldots+\tilde{K}_{r}\left(x_{r}, y_{r}\right)$.

It can be shown that the function $\underline{H}$ of Theorem 3 can be expressed alternatively as

$$
\underline{H}(x, y)=\sup _{\Sigma x_{i}=x ; x_{i} \in C_{i}} \liminf _{y^{\prime} \rightarrow y} \inf _{\sum y_{i}=y^{\prime}} \sum \tilde{K}_{i}\left(x_{i}, y_{i}\right)
$$

whenever $y \in \operatorname{dom}_{2} K_{1}+\ldots+\operatorname{dom}_{2} K_{r}$, and similarly

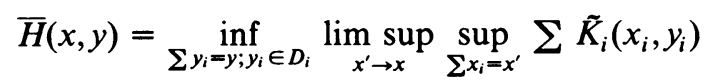

whenever $x \in \operatorname{dom}_{1} K_{1}+\ldots+\operatorname{dom}_{1} K_{r}$. If the sets dom $K_{i}$ are all bounded, these representations for $\underline{H}$ and $\bar{H}$ hold for all $(x, y)$.

Our second dual pair of operations involves combining an equivalence class with a product linear transformation, i.e. a transformation of the form $A(u, v)$ $=\left(A_{1} u, A_{2} v\right)$, where $A_{1}$ and $A_{2}$ are each linear transformations. We say that such an $A$ is a mapping of $R^{p} \times R^{q}$ into $R^{m} \times R^{n}$ if and only if $A_{1}$ maps $R^{p}$ into $R^{m}$ and $A_{2}$ maps $R^{q}$ into $R^{n}$. Trivially, the range of such an $A$ satisfies range $A$ $=$ range $A_{1} \times$ range $A_{2}$, and the adjoint satisfies $A^{*}\left(u^{*}, v^{*}\right)=\left(A_{1}^{*} u^{*}, A_{2}^{*} v^{*}\right)$.

Suppose $[K]$ is an equivalence class of concave-convex functions on $R^{p} \times R^{q}$ and $A$ is a product linear transformation mapping $R^{m} \times R^{n}$ into $R^{p} \times R^{q}$. We say that $[K A]$ is well-defined if and only if all the concave-convex functions of the form

$$
(u, v) \rightarrow \tilde{K} A(u, v)=\tilde{K}(A(u, v)),
$$

for $\tilde{K} \in[K]$, belong to a single equivalence class, in which case $[K A]$ denotes this equivalence class. The operation which sends $[K]$ into $[K A]$ is called forming the composition of $[K]$ with $A$. Our results concerning this operation are collected in the next theorem.

Theorem 4. Let $[K]$ be an equivalence class of closed proper concave-convex functions on $R^{p} \times R^{q}$, let $A$ be a product linear transformation mapping $R^{m} \times R^{n}$. 
into $R^{p} \times R^{q}$, and assume range $A \cap \operatorname{ri}(\operatorname{dom} K) \neq \varnothing$. Then

(i) $[K A]$ is well-defined, closed and proper with $\operatorname{dom}(K A)=A^{-1} \operatorname{dom} K$;

(ii) the least and greatest elements of $[K A]$ are the functions $\underline{K} A$ and $\bar{K} A$, respectively; and

(iii) the subdifferential of $[K A]$ is given by

$$
\partial(K A)(u, v)=A^{*} \partial K(A(u, v)) \text {. }
$$

For our last operation, suppose again that $[K]$ is an equivalence class of concave-convex functions on $R^{p} \times R^{q}$ but that now $A$ is a product linear transformation mapping $R^{p} \times R^{q}$ into $R^{m} \times R^{n}$. We say that $[A K]$ is well-defined if and only if all the concave-convex functions of the form

$$
(u, v) \rightarrow \sup _{\left\{x \mid A_{1} x=u\right\}} \inf _{\left\{y \mid A_{2} y=v\right\}} \tilde{K}(x, y)
$$

or of the form

$$
(u, v) \rightarrow \inf _{\left\{y \mid A_{2} y=v\right\}} \sup _{\left\{x \mid A_{1} x=u\right\}} \tilde{K}(x, y),
$$

for $\tilde{K} \in[K]$, belong to a single equivalence class, in which case $[A K]$ denotes this equivalence class. The operation which sends $[K]$ into $[A K]$ is called taking the image of $[K]$ under $A$. Notice that the elements of $[A K]$ having the form above describe, as a function of $(u, v)$, the lower and upper saddle values in the constrained saddle point problem, "find the saddle points of $\tilde{K}$ with respect to $A_{1}^{-1} u \times A_{2}^{-1} v$." The next two theorems can thus be viewed as assertions about this parametrized class of constrained saddle point problems, as well as about the operation of forming $[A K]$.

Theorem 5. Let $[K]$ be an equivalence class of closed proper concave-convex functions on $R^{p} \times R^{q}$, let $A$ be a product linear transformation mapping $R^{p} \times R^{q}$ into $R^{m} \times R^{n}$, and assume range $A^{*} \cap \operatorname{ri}\left(\operatorname{dom} K^{*}\right) \neq \varnothing$. Then $[A K]$ is well-defined, closed and proper with $\operatorname{dom}(A K) \subset A \operatorname{dom} K$. Moreover, $\left[(A K)^{*}\right]=\left[K^{*} A^{*}\right]$.

There are examples to show that, for arbitrary closed proper equivalence classes, these results are in a sense "best possible." For instance, $[K A]$ and $\left[A^{*} K^{*}\right]$ can fail to exist (i.e. fail to be well-defined) if the intersection condition used in Theorems 4 and 5 is relaxed even slightly. To see this, let $A$ be the zero product transformation $(u, v) \rightarrow(0,0)$ mapping $R^{1} \times R^{1}$ into itself, and let $[K]$ be the closed proper equivalence class whose kernel is the function $(x, y) \rightarrow x^{y}$ defined on the open unit square. (This $[K]$ is discussed in [10, p. 360] and [11, p. 115].) Then range $A \cap \operatorname{ri}(\operatorname{dom} K)=\varnothing$, but one still has range $A \cap \operatorname{dom} K \neq \varnothing$. However it is easy to see that, as $\tilde{K}$ varies over $[K]$, the functions $\tilde{K} A$ determine $2^{\aleph_{0}}$ distinct closed proper equivalence classes (cf. Theorem 4). Moreover, even though $\left[K^{*}\right]$ consists of only one saddle function and the functions 


$$
\left(u^{*}, v^{*}\right) \rightarrow \sup _{\left\{x^{*} \mid A x^{*}=u^{*}\right\}} \inf _{\left\{y^{*} \mid A \xi y^{*}=v^{*}\right\}} K^{*}\left(x^{*}, y^{*}\right)
$$

and

$$
\left(u^{*}, v^{*}\right) \rightarrow \inf _{\left\{y^{*} \mid A^{*} y^{*}=v^{*}\right\}} \sup _{\left\{x^{*}|A| x^{*}=u^{*}\right\}} K^{*}\left(x^{*}, y^{*}\right)
$$

are each closed and proper, they are not equivalent (cf. Theorem 5 ).

This example can be modified to show something more. Let $[K]$ and $A_{1}$ be as before, but now let $A_{2}$ be the identity transformation on $R^{1}$. Then range $A_{1}$ $\cap \operatorname{dom}_{1} K \neq \varnothing$, range $A_{2} \cap \operatorname{ri}\left(\operatorname{dom}_{2} K\right) \neq \varnothing$, and the functions $\tilde{K} A$ still determine $2^{\aleph_{0}}$ distinct proper equivalence classes as $\tilde{K}$ varies over $[K]$. But this time only one of these equivalence classes is closed, the one containing $K A$.

Theorems 4 and 5 together yield a single, simple condition under which $[A K]$ and $\left[K^{*} A^{*}\right]$ are well-defined closed proper equivalence classes conjugate to each other. In particular, the formula $\left[(A K)^{*}\right]=\left[K^{*} A^{*}\right]$ means that each of our second pair of operations is in fact the dual of the other with respect to the conjugacy correspondence.

Notice that the subdifferential of $[A K]$ can be characterized using the conjugacy formula of Theorem 5 and the subdifferential formula of Theorem 4 .

It is trivial to show that the effective domain inclusion of Theorem 5 can be strengthened to equality when $A$ is one-to-one. But unfortunately the $A$ 's in which one is usually interested (e.g. projections or addition transformations) are not one-to-one, and in this event examples can be given which exhibit the "collapsing" behavior discussed above for extremal convolution. However by imposing growth conditions on $K$ or $K^{*}$ one can ensure that $\operatorname{dom}(A K)$ $=A$ dom $K$. For instance (under the hypotheses of Theorem 5), $R^{m} \times R^{n}$ $=\operatorname{dom}(A K)=A \operatorname{dom} K$ when $A^{*-1} \operatorname{dom} K^{*}$ is bounded.

The extrema appearing in the definition of $[A K]$ are usually attained in a very strong, stable sense, as explained in the next theorem. For the statement of this theorem, recall the definition of the image $B h$ of a convex or concave function $h$ by a linear transformation $B:$ If $h$ is convex, then $(B h)(w)=\inf \{h(z) \mid B z=w\}$, and if $h$ is concave, then $(B h)(w)=\sup \{h(z) \mid B z=w\}$.

Theorem 6. Let $[K]$ and $A$ be as in Theorem 5 , and let $\tilde{K} \in[K]$. Then the least element of $[A K]$ is the function

$$
\underline{J}(u, v)=\sup _{\left\{x \mid A_{1} x=u\right\}} \operatorname{cl}\left(A_{2} \tilde{K}(x, \cdot)\right)(v)
$$

where $\left\{x \mid A_{1} x=u\right\}$ can be replaced by $\left\{x \in \operatorname{dom}_{1} K \mid A_{1} x=u\right\}$ for every $u$, and similarly the greatest element of $[A K]$ is the function

$$
\bar{J}(u, v)=\inf _{\left\{y \mid A_{2} y=v\right\}} \operatorname{cl}\left(A_{1} \tilde{K}(\cdot, y)\right)(u),
$$

where $\left\{y \mid A_{2} y=v\right\}$ can be replaced by $\left\{y \in \operatorname{dom}_{2} K \mid A_{2} y=v\right\}$ for every $v$. Moreover, for each $(u, v) \in \operatorname{dom} \partial(A K)$ the extrema appearing in the definitions of 
$\underline{J}(u, v)$ and $\bar{J}(u, v)$ are attained, in the sense that there exists a pair $(x, y) \in \operatorname{dom} \partial K$ such that $A(x, y)=(u, v)$ and the quantities

$$
\underline{J}(u, v)=\operatorname{cl}\left(A_{2} \tilde{K}(x, \cdot)\right)(v)=\liminf _{v^{\prime} \rightarrow v}\left\{\inf _{y^{\prime} \in A_{2}^{-1} v^{\prime}} \tilde{K}\left(x, y^{\prime}\right)\right\}
$$

and

$$
\left.\bar{J}(u, v)=\operatorname{cl}\left(A_{1} \tilde{K}(\cdot, y)\right)(u)=\lim _{u^{\prime} \rightarrow u} \sup _{x^{\prime} \in A_{1}^{-1} u^{\prime}} \tilde{K}\left(x^{\prime}, y\right)\right\}
$$

are finite and equal to $\tilde{K}(x, y)$.

Regarding the expressions for $\underline{J}$ given in the first part of Theorem 6 , one has

$$
\operatorname{cl}\left(A_{2} \tilde{K}(x, \cdot)\right)(v)=\liminf _{v^{\prime} \rightarrow v}\left\{\inf _{y \in A_{2}^{-1} v^{\prime}} \tilde{K}(x, y)\right\}
$$

whenever $v \in A_{2} \operatorname{dom}_{2} K$ or whenever $A_{2} \tilde{K}(x, \cdot)$ is never $-\infty$ (e.g. if $x \in \operatorname{dom}_{1} K$ and $\operatorname{dom}_{2} K$ is bounded). Similarly, regarding $\bar{J}$ one has

$$
\left.\operatorname{cl}\left(A_{1} \tilde{K}(\cdot, y)\right)(u)=\lim _{u^{\prime} \rightarrow u} \sup _{x \in A_{1}^{-1} u^{\prime}} \tilde{K}(x, y)\right\}
$$

whenever $u \in A_{1} \operatorname{dom}_{1} K$ or whenever $A_{1} \tilde{K}(\cdot, y)$ is never $+\infty$ (e.g. if $y \in \operatorname{dom}_{2} K$ and $\operatorname{dom}_{1} K$ is bounded).

Observe that the pair $(x, y)$ whose existence is guaranteed in Theorem 6 is a fortiori a saddle point of $\tilde{K}$ with respect to $A_{1}^{-1} u \times A_{2}^{-1} v$. Moreover the string of equalities which $(x, y)$ actually satisfies can be viewed as a type of stability property quite similar to the ones dealt with by Rockafellar [7], [8]. In the presence of $A(x, y)=(u, v)$ it can be shown that these equalities are equivalent to the condition that for every $\epsilon>0$ there exists a $\delta>0$ such that $\left\|u^{\prime}-u\right\| \leq \delta$ and $\left\|v^{\prime}-v\right\| \leq \delta$ imply

$$
\alpha-\epsilon \leq \inf _{A_{2}^{-1} v^{\prime}} \tilde{K}(x, \cdot) \quad \text { and } \quad \sup _{A_{1}^{-1} \mathcal{u}^{\prime}} \tilde{K}(\cdot, y) \leq \alpha+\epsilon,
$$

where $\alpha=\tilde{K}(x, y) \in R$.

From the results of Theorems 5 and 6 one can easily deduce a strong duality theorem for a certain pair of saddle point problems. Let $K$ and $A$ be as in Theorems 5 and 6 , and let $(u, v) \in \operatorname{dom} \partial(A K)$ be fixed. By Theorem 6, there exists a pair $(x, y) \in \operatorname{dom} \partial K$ which is a saddle point of $\tilde{K}$ with respect to $A_{1}^{-1} u \times A_{2}^{-1} v$. But also, for any pair $\left(u^{*}, v^{*}\right) \in \partial(A K)(u, v)$, the duality formula $\left[(A K)^{*}\right]=\left[K^{*} A^{*}\right]$ together with Theorems 37.5 and 37.4 of [10] imply that $\left(u^{*}, v^{*}\right)$ is a saddle point of the convex-concave function $\langle\cdot, u\rangle+\langle\cdot, v\rangle-\tilde{K}^{*} A^{*}$ with respect to $R^{m} \times R^{n}$. And furthermore, from $[A K]=\left[\left(K^{*} A^{*}\right)^{*}\right]$ it follows that the saddle values in these two saddle point problems are equal. Thus, we have 
that

$$
\operatorname{maximin}_{A_{1}^{-1} u \times A_{2}^{-1} v}\{\tilde{K}\}=\underset{R^{m} \times R^{n}}{\operatorname{minimax}}\left\{\langle\cdot, u\rangle+\langle\cdot, v\rangle-\tilde{K}^{*} A^{*}\right\},
$$

and the pairs $(x, y)$ and $\left(u^{*}, v^{*}\right)$ are solutions, respectively.

By now the reader may already have observed the close parallel between the results for $\left[K_{1}+\ldots+K_{r}\right]$ and $\left[K_{1} \square \ldots \square K_{r}\right]$ and those for $[K A]$ and $[A K]$. While the proofs of the corresponding results can likewise be carried out in parallel fashion, we choose to avoid presenting such essentially repetitive proofs. Instead, our approach is to develop some additional material on the basis of which Theorems 1, 2 and 3 follow as immediate corollaries of Theorems 4, 5 and 6 , respectively. This additional material involves extending to saddle functions the concept of a separable convex function, and then proving the basic facts concerning "separable" saddle functions. We should mention that this does involve some subtleties, and that the proof of Theorem 7 (notably part (iv)) is nontrivial.

Suppose for each $i=1, \ldots, r$ that $\left[K_{i}\right]$ is an equivalence class of concaveconvex functions on $R^{m_{i}} \times R^{n_{i}}$. Write $C=\operatorname{dom}_{1} K_{1} \times \ldots \times \operatorname{dom}_{1} K_{r}, \quad D$ $=\operatorname{dom}_{2} K_{1} \times \ldots \times \operatorname{dom}_{2} K_{r}, m=m_{1}+\ldots+m_{r}, n=n_{1}+\ldots+n_{r}$, and let the points of $R^{m}$ and $R^{n}$ be written as $x=\left(x_{1}, \ldots, x_{r}\right)$ and $y=\left(y_{1}, \ldots, y_{r}\right)$, respectively. We say that $\left[\left(K_{1}, \ldots, K_{r}\right)\right]$ is well-defined if and only if all the concave-convex functions of the form

$$
\begin{aligned}
(x, y) & \rightarrow \tilde{K}_{1}\left(x_{1}, y_{1}\right)+\ldots+\tilde{K}_{r}\left(x_{r}, y_{r}\right) & & \text { if } x \in C, \\
& \rightarrow-\infty & & \text { if } x \notin C,
\end{aligned}
$$

or of the form

$$
\begin{array}{rlrl}
(x, y) & \rightarrow \tilde{K}_{1}\left(x_{1}, y_{1}\right)+\ldots+\tilde{K}_{r}\left(x_{r}, y_{r}\right) & & \text { if } y \in D, \\
& \rightarrow+\infty & \text { if } y \notin D,
\end{array}
$$

for $\tilde{K}_{1} \in\left[K_{1}\right], \ldots, \tilde{K}_{r} \in\left[K_{r}\right]$, belong to a single equivalence class, in which case $\left[\left(K_{1}, \ldots, K_{r}\right)\right]$ is this equivalence class. Note that this definition avoids the dilemma of having to add $+\infty$ to $-\infty$. Our final theorem is the following.

Theorem 7. Let the $\left[K_{i}\right]$ and various notations be as in the definition above, and assume that each $\left[K_{i}\right]$ is closed and proper. Then

(i) $\left[\left(K_{1}, \ldots, K_{r}\right)\right]$ is well-defined, closed and proper with

$$
\operatorname{dom}\left(K_{1}, \ldots, K_{r}\right)=C \times D ;
$$

(ii) the least and greatest elements of $\left[\left(K_{1}, \ldots, K_{r}\right)\right]$ are the functions

$$
\begin{aligned}
\underline{K}(x, y) & =\underline{K}_{1}\left(x_{1}, y_{1}\right)+\ldots+\underline{K}_{r}\left(x_{r}, y_{r}\right) & & \text { if } x \in C, \\
& =-\infty & & \text { if } x \notin C,
\end{aligned}
$$


and

$$
\begin{aligned}
\bar{K}(x, y) & =\bar{K}_{1}\left(x_{1}, y_{1}\right)+\ldots+\bar{K}_{r}\left(x_{r}, y_{r}\right) & & \text { if } y \in D, \\
& =+\infty & & \text { if } y \notin D,
\end{aligned}
$$

respectively;

(iii) for $j=1$ and 2 and $(x, y) \in C \times D$,

$$
\partial_{j}\left(K_{1}, \ldots, K_{r}\right)(x, y)=\partial_{j} K_{1}\left(x_{1}, y_{1}\right) \times \ldots \times \partial_{j} K_{r}\left(x_{r}, y_{r}\right)
$$

while $\partial\left(K_{1}, \ldots, K_{r}\right)(x, y)=\varnothing$ when $(x, y) \notin C \times D$; and

(iv) $\left[\left(K_{1}, \ldots, K_{r}\right)^{*}\right]=\left[\left(K_{1}^{*}, \ldots, K_{r}^{*}\right)\right]$.

2. Proofs. The plan is first to prove Theorems 4 through 7 . Then Theorem 7 will be combined with each of Theorems 4, 5 and 6 to yield Theorems 1, 2 and 3 , respectively, as corollaries.

In carrying out the proofs we shall cite results from Rockafellar [10] on numerous occasions. For brevity, therefore, we adopt the convention of suppressing explicit reference to [10] and citing such results by merely enclosing their numbers in parentheses. For example, Theorem 34.2 of [10] is cited simply as (34.2), Corollary 37.4.1 as (37.4.1), and so on.

Although the fact was hinted at as we went along, we should point out explicitly that in defining the four operations and separable saddle functions above, in each case the functions referred to in those definitions are indeed concave-convex when the $K$ or $K_{i}$ 's are. This can be shown with the aid of (5.2), (5.5) and (5.7).

Proof of Theorem 4. Write $\operatorname{dom} K=C \times D$ and let $\tilde{K} \in[K]$. For each $u \in A_{1}^{-1} C, \tilde{K}\left(A_{1} u, \cdot\right)$ is never $-\infty$ and hence $\tilde{K} A(u, \cdot)=\tilde{K}\left(A_{1} u, \cdot\right) A_{2}$ is never $-\infty$. Thus $A_{1}^{-1} C \subset \operatorname{dom}_{1}(\tilde{K} A)$, and similarly $A_{2}^{-1} D \subset \operatorname{dom}_{2}(\tilde{K} A)$. Now suppose $u \notin A_{1}^{-1} C$. Since $\tilde{K}$ is closed and proper, (34.3) implies $\tilde{K}\left(A_{1} u, \cdot\right) A_{2}$ equals $-\infty$ everywhere on ri $D$ and hence $\tilde{K} A(u, \cdot)$ equals $-\infty$ everywhere on $A_{2}^{-1}$ (ri $\left.D\right)$. Since $A_{2}^{-1}$ (ri $\left.D\right) \neq \varnothing$ by hypothesis, this shows $u \notin \operatorname{dom}_{1}(\tilde{K} A)$. Thus $A_{1}^{-1} C$ $=\operatorname{dom}_{1}(\tilde{K} A)$ actually holds, and similarly $A_{2}^{-1} D=\operatorname{dom}_{2}(\tilde{K} A)$. In particular, this means that $\underline{K} A$ and $\bar{K} A$ are each proper concave-convex functions having effective domain $A^{-1}$ (dom $K$ ).

Now observe from (34.2) that a closed saddle function is convex-closed (resp. concave-closed) if and only if it is the least (resp. greatest) element of its equivalence class. It is routine to show, using (6.7), (34.3) and (9.5), that $\underline{K A}$ satisfies the six conditions of (34.3) and moreover is convex-closed. Therefore $\underline{K} A$ is both closed and the least element of its equivalence class, and similarly $\bar{K} A$ is closed and the greatest element of its equivalence class.

According to (37.4), two closed proper saddle functions are equivalent if and only if they have the same kernel. But for any $(u, v) \in \operatorname{ri}\left(A^{-1} \operatorname{dom} K\right)$, it follows from the hypothesis and (6.7) that $A(u, v) \in \operatorname{ri}(\operatorname{dom} K)$, so that $\underline{K} \sim \bar{K}$ implies 
$\underline{K} A(u, v)=\bar{K} A(u, v)$. Thus $\underline{K} A$ and $\bar{K} A$ have the same kernel, and so they belong to the same equivalence class, call it $[H]$. Now let $\tilde{K}$ be any element of $[K]$. Then $\underline{K} \leq \tilde{K} \leq \bar{K}$ by (34.2), and hence $\underline{K} A \leq \tilde{K} A \leq \bar{K} A$. From this it follows trivially that $\tilde{K} A \in[H]$. This shows that $[K A]$ is well-defined and is just the class $[H]$, thus completing the proof of everything except the subdifferential formula.

By (37.4), for any closed proper saddle function $K$ one has dom $\partial K \subset \operatorname{dom} K$. This together with $\operatorname{dom}(K A)=A^{-1} \operatorname{dom} K$ implies that $\partial(K A)(u, v)=\varnothing$ $=A^{*} \partial K(A(u, v))$ whenever $(u, v) \notin A^{-1} \operatorname{dom} K$. So suppose $(u, v) \in A^{-1} \operatorname{dom} K$. By the definitions, $\left(u^{*}, v^{*}\right) \in \partial(K A)(u, v)$ if and only if $u^{*} \in \partial\left(K\left(\cdot, A_{2} v\right) A_{1}\right)(u)$ and $v^{*} \in \partial\left(K\left(A_{1} u, \cdot\right) A_{2}\right)(v)$. From (34.3), (6.3.1) and $A_{1} u \in C$ we know that $K\left(A_{1} u, \cdot\right)$ is proper convex with ri(dom $\left.K\left(A_{1} u, \cdot\right)\right)=$ ri $D$. Hence the hypothesis range $A_{2} \cap$ ri $D \neq \varnothing$ and (23.9) imply that $v^{*} \in \partial\left(K\left(A_{1} u, \cdot\right) A_{2}\right)(v)$ if and only if $v^{*} \in A_{2}^{*} \partial K\left(A_{1} u, \cdot\right)\left(A_{2} v\right)$, i.e. if and only if $v^{*} \in A_{2}^{*} \partial_{2} K(A(u, v))$. Similarly, $u^{*} \in \partial\left(K\left(\cdot, A_{2} v\right) A_{1}\right)(u)$ if and only if $u^{*} \in A_{1}^{*} \partial_{1} K(A(u, v))$. The subdifferential formula follows, and Theorem 4 is proved.

For greater clarity in the proof of Theorems 5 and 6, it is helpful to establish the following technical lemma separately.

Lemma 1. Let $[K]$ and $A$ be as in Theorem 5 , and let $\tilde{K} \in[K]$. Then the lower conjugate of $\bar{K}^{*} A^{*}$ is the function

$$
\underline{J}(u, v)=\sup _{\left\{x \mid A_{1} x=u\right\}} \operatorname{cl}\left(A_{2} \tilde{K}(x, \cdot)\right)(v)
$$

where $\left\{x \mid A_{1} x=u\right\}$ can be replaced by $\left\{x \in \operatorname{dom}_{1} K \mid A_{1} x=u\right\}$ for any $u \in R^{m}$, and

$$
\operatorname{cl}\left(A_{2} \tilde{K}(x, \cdot)\right)(v)=\liminf _{v \rightarrow v} A_{2} \tilde{K}(x, \cdot)\left(v^{\prime}\right)
$$

whenever $v \in A_{2} \operatorname{dom}_{2} K$. Similarly, the upper conjugate of $\underline{K}^{*} A^{*}$ is the function

$$
\bar{J}(u, v)=\inf _{\left\{y \mid A_{2} y=v\right\}} \operatorname{cl}\left(A_{1} \tilde{K}(\cdot, y)\right)(u)
$$

where $\left\{y \mid A_{2} y=v\right\}$ can be replaced by $\left\{y \in \operatorname{dom}_{2} K \mid A_{2} y=v\right\}$ for any $v \in R^{n}$, and

$$
\operatorname{cl}\left(A_{1} \tilde{K}(\cdot, y)\right)(u)=\lim _{u^{\prime} \rightarrow u} A_{1} \tilde{K}(\cdot, y)\left(u^{\prime}\right)
$$

whenever $u \in A_{1} \operatorname{dom}_{1} K$.

Proof of Lemma 1. We prove only the first assertion, as the second is similar. Let $\underline{J}$ denote the lower conjugate of $\bar{K}^{*} A^{*}$. Then

$$
\underline{J}(u, v)=\sup _{v^{*}}\left\{\left\langle v^{*}, v\right\rangle+\inf _{u^{*}}\left\{\left\langle u^{*}, u\right\rangle-\left(\bar{K}^{*}\left(\cdot, A_{2}^{*} v^{*}\right) A_{1}^{*}\right)\left(u^{*}\right)\right\}\right\} .
$$

Since $\bar{K}^{*}$ is concave-closed, (34.3) and (6.3.1) imply that ri(dom $\bar{K}^{*}\left(\cdot, y^{*}\right)$ ) equals $\operatorname{ri}\left(\operatorname{dom}_{1} K^{*}\right)$ when $y^{*} \in \operatorname{dom}_{2} K^{*}$ and equals $R^{q}$ when $y^{*} \notin \operatorname{dom}_{2} K^{*}$. Hence (16.3) and the hypothesis range $A_{1}^{*} \cap \operatorname{ri}\left(\operatorname{dom}_{1} K^{*}\right) \neq \varnothing$ imply

$$
\left(\bar{K}^{*}\left(\cdot, A_{2}^{*} v^{*}\right) A_{1}^{*}\right)^{*}(u)=\left(A_{1} \bar{K}^{*}\left(\cdot, A_{2}^{*} v^{*}\right)^{*}\right)(u)=\sup \left\{\underline{g}\left(x, A_{2}^{*} v^{*}\right) \mid A_{1} x=u\right\}
$$


for every $v^{*} \in R^{n}$ and $u \in R^{m}$, where $g\left(x, y^{*}\right)=\inf _{x^{*}}\left\{\left\langle x^{*}, x\right\rangle-\bar{K}^{*}\left(x^{*}, y^{*}\right)\right\}$. Thus,

$$
\begin{aligned}
\underline{J}(u, v) & =\sup _{v^{*}}\left\{\left\langle v^{*}, v\right\rangle+\sup _{x \in A_{1}^{-1} u} g\left(x, A_{2}^{*} v^{*}\right)\right\} \\
& =\sup _{x \in A_{1}^{-1} u} \sup _{v^{*}}\left\{\left\langle v^{*}, v\right\rangle-(-g)\left(x, A_{2}^{*} v^{*}\right)\right\} .
\end{aligned}
$$

But it follows from (34.2) and (37.1) that $-g=f$, where

$$
f\left(x y^{*}\right)=\sup _{y}\left\{\left\langle y, y^{*}\right\rangle-K(x, y)\right\} \text {. }
$$

Indeed, if we use the notation of (34.2) and let $F$ be the unique closed convex bifunction such that $[K]=\Omega(F)$, then $\left[K^{*}\right]=\Omega\left(F_{*}\right)$ by $(37.1)$, and

$$
-g\left(x, y^{*}\right)=-\left(F_{*} y^{*}\right)(x)=(F x)\left(y_{*}\right)=f\left(x, y^{*}\right) .
$$

Therefore (16.3) and (34.2) imply

$$
\begin{aligned}
\sup _{v^{*}}\left\{\left\langle v^{*}, v\right\rangle-(-g)\left(x, A_{2}^{*} v^{*}\right)\right\} & =\left(f(x, \cdot) A_{2}^{*}\right)^{*}(v)=\operatorname{cl}\left(A_{2} f(x, \cdot)^{*}\right)(v) \\
\cdot & =\operatorname{cl}\left(A_{2} \underline{K}(x, \cdot)\right)(v) .
\end{aligned}
$$

This establishes that

$$
\underline{J}(u, v)=\sup _{\left\{x \mid A_{1} x=u\right\}} \operatorname{cl}\left(A_{2} \underline{K}(x, \cdot)\right)(v) .
$$

Next we claim that, for any $\tilde{K} \in[K], \operatorname{cl}\left(A_{2} \tilde{K}(x, \cdot)\right)$ is the constant function $-\infty$ when $x \notin \operatorname{dom}_{1} K$ and $\operatorname{cl}\left(A_{2} \tilde{K}(x, \cdot)\right)=\operatorname{cl}\left(A_{2} \underline{K}(x, \cdot)\right)$ when $x \in \operatorname{dom}_{1} K$. Indeed, if $x \notin \operatorname{dom}_{1} K$, then (34.3) implies that $\tilde{K}(x, \cdot)$ equals $-\infty$ on ri(dom $\left.{ }_{2} K\right)$, so that $A_{2} \tilde{K}(x, \cdot)$ equals $-\infty$ on the (nonempty) set $A_{2}$ ri( $\left(\operatorname{dom}_{2} K\right)$, and hence $\operatorname{cl}\left(A_{2} \tilde{K}(x, \cdot)\right)$ is identically $-\infty$ by definition. On the other hand, suppose $x \in \operatorname{dom}_{1} K$ and put $h=\tilde{K}(x, \cdot)$. Then $\mathrm{cl} h=\operatorname{cl}_{2} \tilde{K}(x, \cdot)=\underline{K}(x, \cdot)$ by (34.2), so we are actually claiming that $\operatorname{cl}\left(A_{2} h\right)=\operatorname{cl}\left(A_{2}(\mathrm{cl} h)\right)$. By (7.3.4) this will follow once we know that ri(dom $\left.A_{2} h\right)=\operatorname{ri}\left(\operatorname{dom} A_{2}(\operatorname{cl} h)\right)$ and that the functions $A_{2} h$ and $A_{2}(\mathrm{cl} h)$ agree on this set. Clearly $\operatorname{dom} A_{2} h=A_{2} D_{1}$ and $\operatorname{dom} A_{2}(\mathrm{cl} h)$ $=A_{2} D_{2}$, where $D_{1}=\operatorname{dom} h$ and $D_{2}=\operatorname{dom}(\operatorname{cl} h)$. Since $x \in \operatorname{dom}_{1} K$, (34.3) implies that $h$ is proper convex and hence $D_{1} \subset D_{2} \subset \mathrm{cl} D_{1}$. By (6.3.1) and (6.6) it follows that $\operatorname{ri}\left(A_{2} D_{1}\right)=\operatorname{ri}\left(A_{2} D_{2}\right)$. Since $h$ is convex, $\operatorname{cl} h \leq h$ whence $A_{2}(\mathrm{cl} h)$ $\leq A_{2} h$. To show the reverse inequality for $v \in \operatorname{ri}\left(A_{2} D_{1}\right)$, pick some $\bar{y} \in A_{2}^{-1} v$ $\cap$ ri $D_{1}$. Then for any $y \in A_{2}^{-1} v,(7.5)$ together with the properness of $h$ and the convexity of $A_{2}^{-1} v$ imply that

$$
(\operatorname{cl} h)(y)=\lim _{\lambda \uparrow 1} h\left(y_{\lambda}\right) \geq \inf _{0 \leq \lambda<1} h\left(y_{\lambda}\right) \geq\left(A_{2} h\right)(v),
$$

where $y_{\lambda}=(1-\lambda) \bar{y}+\lambda y$. Thus $A_{2}(\mathrm{cl} h) \geq A_{2} h$ on $\operatorname{ri}\left(A_{2} D_{1}\right)$, and the claim is established. 
By the claim and the representation already obtained for $\underline{J}$, it follows immediately that

$$
\underline{J}(u, v)=\sup _{\left\{x \mid A_{1} x=u\right\}} \operatorname{cl}\left(A_{2} \tilde{K}(x, \cdot)\right)(v) .
$$

Furthermore, in view of the convention sup $\varnothing=-\infty$, when taking a supremum we can omit those elements which yield the value $-\infty$. Since $\operatorname{cl}\left(A_{2} \tilde{K}(x, \cdot)\right)$ is constantly $-\infty$ whenever $x \notin \operatorname{dom}_{1} K$, this means that in the representation just given for $\underline{J}$ the set $\left\{x \mid A_{1} x=u\right\}$ can be replaced by $\left\{x \in \operatorname{dom}_{1} K \mid A_{1} x=u\right\}$.

Finally, we need to show that $(\operatorname{cl} h)(v)=\lim _{\inf _{v^{\prime} \rightarrow v}} h\left(v^{\prime}\right)$ whenever $v$ $\in A_{2} \operatorname{dom}_{2} K$, where $h=A_{2} \tilde{K}(x, \cdot)$. From the nature of the closure operation for convex functions, we know this equality holds unless $(\mathrm{cl} h)(v)=-\infty$ and $\lim \inf _{v^{\prime} \rightarrow v} h\left(v^{\prime}\right)=+\infty$. Now (34.3) implies that $\operatorname{dom}_{2} K \subset \operatorname{dom} \tilde{K}(x, \cdot)$, so that $A_{2} \operatorname{dom}_{2} K \subset \operatorname{dom} h$. Hence $v \in A_{2} \operatorname{dom}_{2} K$ implies $\lim \inf _{v \rightarrow v} h\left(v^{\prime}\right) \leq h(v)$ $<+\infty$. This completes the proof of Lemma 1 .

Joint proof of Theorems 5 and 6. The hypotheses imply that Theorem 4 applies to $\left[K^{*}\right]$ and $A^{*}$. By (37.1.1) and (34.2.2), the functions $\underline{J}$ and $\bar{J}$ in Lemma 1 are the least and greatest elements of the equivalence class conjugate to $\left[K^{*} A^{*}\right]$. Let $\tilde{K}$ be any element of $[K]$. Since $\operatorname{cl} f \leq f$ when $f$ is convex, $g \leq \operatorname{cl} g$ when $g$ is concave, and $\sup _{S} \inf _{T} H \leq \inf _{T} \sup _{S} H$ for an arbitrary function $H$ on $S \times T$, it follows that

$$
\underline{J}(u, v) \leq \sup _{\left\{x \mid A_{1} x=u\right\}} \inf _{\left\{y \mid A_{2} y=v\right\}} \tilde{K}(x, y) \leq \inf _{\left\{y \mid A_{2} y=v\right\}} \sup _{\left\{x \mid A_{1} x=u\right\}} \tilde{K}(x, y) \leq \bar{J}(u, v)
$$

for every $(u, v)$. By (34.2) this shows that $[A K]$ is well-defined and coincides with the equivalence class conjugate to $\left[K^{*} A^{*}\right]$. Since the conjugacy correspondence among closed proper equivalence classes is one-to-one and symmetric, this shows that $[A K]$ is closed and proper and satisfies $\left[(A K)^{*}\right]=\left[K^{*} A^{*}\right]$.

To see that $\operatorname{dom}(A K) \subset A \operatorname{dom} K$, notice first that $\operatorname{dom}(A K)=\operatorname{dom}_{1} J_{1}$ $\times \operatorname{dom}_{2} J_{2}$ for any two elements $J_{1}$ and $J_{2}$ of $[A K]$. Since the functions

$$
J_{1}(u, v)=\sup _{\left\{x \in \operatorname{dom}|K| A_{1} x=u\right\}} \inf _{\left\{y \mid A_{2} y=v\right\}} \tilde{K}(x, y)
$$

and

$$
J_{2}(u, v)=\inf _{\left\{y \in \operatorname{dom}_{2} K \mid A_{2} y=v\right\}} \sup _{\left\{x \mid A_{1} x=u\right\}} \tilde{K}(x, y)
$$

are concave-convex (by (5.5) and (5.7)) and satisfy $\underline{J} \leq J_{1} \leq J_{2} \leq \bar{J}$ by Lemma 1 , they belong to $[A K]$. But the conventions sup $\varnothing=-\infty$ and inf $\varnothing=+\infty$ imply trivially that $\operatorname{dom}_{1} J_{1} \subset A_{1} \operatorname{dom}_{1} K$ and $\operatorname{dom}_{2} J_{2} \subset A_{2} \operatorname{dom}_{2} K$. Hence $\operatorname{dom}(A K) \subset A \operatorname{dom} K$. This completes the proof of Theorems 5 and 6 except for the attainment assertion of Theorem 6.

Now let $(u, v) \in \operatorname{dom} \partial(A K)$ be given. Then $\left(u^{*}, v^{*}\right) \in \partial(A K)(u, v)$ for some $\left(u^{*}, v^{*}\right)$, which by $(37.5)$ and the formula $\left[(A K)^{*}\right]=\left[K^{*} A^{*}\right]$ means that $(u, v)$ 
$\in \partial\left(K^{*} A^{*}\right)\left(u^{*}, v^{*}\right)$ for some $\left(u^{*}, v^{*}\right)$. By the subdifferential formula of Theorem 4 together with (37.5), this is equivalent to $A(x, y)=(u, v)$ and $A^{*}\left(u^{*}, v^{*}\right)$ $\in \partial K(x, y)$ for some $(x, y) \in \operatorname{dom} \partial K$ and $\left(u^{*}, v^{*}\right)$. It follows by (37.4) and (37.4.1) that, for any $\tilde{K} \in[K]$,

$$
\begin{aligned}
\tilde{K}\left(x^{\prime}, y\right)-\left\langle x^{\prime}, A_{1}^{*} u^{*}\right\rangle-\left\langle v, v^{*}\right\rangle & \leq \tilde{K}(x, y)-\left\langle u, u^{*}\right\rangle-\left\langle v, v^{*}\right\rangle \\
& \leq \tilde{K}\left(x, y^{\prime}\right)-\left\langle u, u^{*}\right\rangle-\left\langle y^{\prime}, A_{2}^{*} v^{*}\right\rangle
\end{aligned}
$$

for all $\left(x^{\prime}, y^{\prime}\right) \in R^{p} \times R^{q}$. Therefore

$$
A_{1} \tilde{K}(\cdot, y)(u)=\sup _{\left\{x^{\prime} \mid A_{1} x^{\prime}=u\right\}} \tilde{K}\left(x^{\prime}, y\right)=\alpha=\inf _{\left\{y^{\prime} \mid A_{2} y^{\prime}=v\right\}} \tilde{K}\left(x, y^{\prime}\right)=A_{2} \tilde{K}(x, \cdot)(v),
$$

where $\alpha=\tilde{K}(x, y) \in R$. Since

$$
\operatorname{cl}\left(A_{2} \tilde{K}(x, \cdot)\right)(v) \leq \underline{J}(u, v) \leq \bar{J}(u, v) \leq \operatorname{cl}\left(A_{1} \tilde{K}(\cdot, y)\right)(u),
$$

to complete the proof of the attainment assertion it suffices to show for the convex function $h=A_{2} \tilde{K}(x, \cdot)$ that

$$
h(v)=(\operatorname{cl} h)(v)=\liminf _{v \rightarrow v} h\left(v^{\prime}\right)
$$

and for the concave function $k=A_{1} \tilde{K}(\cdot, y)$ that

$$
k(u)=(\operatorname{cl} k)(u)=\lim _{u^{\prime} \rightarrow u} \sup k\left(u^{\prime}\right) .
$$

We only show the equalities for $h$, as those for $k$ are similar. Since $\alpha=h(v)$, the system of inequalities above implies that

$$
\tilde{K}\left(x, y^{\prime}\right) \geq h(v)+\left\langle v^{*}, A_{2} y^{\prime}-v\right\rangle, \quad \forall y^{\prime} \in R^{q},
$$

that is,

$$
\tilde{K}\left(x, y^{\prime}\right) \geq h(v)+\left\langle v^{*}, v^{\prime}-v\right\rangle, \quad \forall y^{\prime} \in A_{2}^{-1} v^{\prime}, \forall v^{\prime} \in \text { range } A_{2} .
$$

Hence

$$
h\left(v^{\prime}\right) \geq h(v)+\left\langle v^{*}, v^{\prime}-v\right\rangle, \quad \forall v^{\prime} \in \operatorname{range} A_{2} .
$$

Since range $A_{2} \supset \operatorname{dom} h$, this means that $v^{*} \in \partial h(v)$. Then since $h$ is finite and subdifferentiable at $v,(23.3)$ implies that $h$ is proper. Thus $(\mathrm{cl} h)(v)$ $=\lim \inf _{v^{\prime} \rightarrow v} h\left(v^{\prime}\right)$. Moreover, since $h$ is proper and subdifferentiable at $v,(23.5 .2)$ implies that $h(v)=(\mathrm{cl} h)(v)$. This completes the joint proof of Theorems 5 and 6 .

To prove Theorem 7 we need some corresponding facts about separable convex functions. These are listed in the following lemma, whose easy proof is left to the reader. (Part (iv) of Lemma 2 is not explicitly used in proving part (iv) of Theorem 7, but we include it to highlight the strong parallel between separable convex and separable saddle functions.) 
Lemma 2. For $i=1, \ldots, r$ let $h_{i}$ be a convex function on $R^{n_{i}}$ which is never $-\infty$, and define $h(y)=h_{1}\left(y_{1}\right)+\ldots+h_{r}\left(y_{r}\right)$ for each $y=\left(y_{1}, \ldots, y_{r}\right) \in R^{n}$, where $n=n_{1}+\ldots+n_{r}$. Then

(i) $h$ is a convex function on $R^{n}$, proper if each $h_{i}$ is proper, and

$$
\operatorname{dom} h=\operatorname{dom} h_{1} \times \ldots \times \operatorname{dom} h_{r} \text {; }
$$

(ii) $(\mathrm{cl} h)(y)=\left(\mathrm{cl} h_{1}\right)\left(y_{1}\right)+\ldots+\left(\mathrm{cl} h_{r}\right)\left(y_{r}\right)$;

(iii) $\partial h(y)=\partial h_{1}\left(y_{1}\right) \times \ldots \times \partial h_{r}\left(y_{r}\right)$; and

(iv) $h^{*}\left(y^{*}\right)=h_{1}^{*}\left(y_{1}^{*}\right)+\ldots+h_{r}^{*}\left(y_{r}^{*}\right)$.

Proof of Theorem 7. To establish (i) and (ii), we first show that the function $\bar{K}$ defined in (ii) is closed, proper concave-convex with effective domain $C \times D$ and moreover is concave-closed. This we do by showing that $\bar{K}$ together with the sets $C$ and $D$ as defined above satisfy the six conditions of (34.3) and moreover that $\bar{K}(\cdot, y)$ is closed for each $y$.

If $y \notin D$ then $\bar{K}(\cdot, y)$ is the constant function $+\infty$, which is trivially concave and closed. Suppose $y \in D$. Since each $\bar{K}_{i}$ is closed, proper and concave-closed, (34.3) implies that each $\bar{K}\left(\cdot, y_{i}\right)$ is closed proper concave with $C_{i} \subset \operatorname{dom} \bar{K}\left(\cdot, y_{i}\right)$ $\subset \operatorname{cl~} C_{i}$, and $C_{i}=\operatorname{dom} \bar{K}\left(\cdot, y_{i}\right)$ when actually $y_{i} \in$ ri $D_{i}$. It follows by (i) and (ii) of Lemma 2 that $\bar{K}(\cdot, y)$ is a closed proper concave function with $C \subset \operatorname{dom} \bar{K}(\cdot, y)$ $\subset \operatorname{cl} C_{1} \times \ldots \times \operatorname{cl} C_{r}=\operatorname{cl} C$, and $C=\operatorname{dom} \bar{K}(\cdot, y)$ when actually $y \in$ ri $D$ $=$ ri $D_{1} \times \ldots \times$ ri $D_{r}$. Now let $x \in R^{m}$ be fixed and put $h_{i}=\bar{K}\left(x_{i}, \cdot\right)$ for $i$ $=1, \ldots, r$. Then

$$
\begin{aligned}
\bar{K}(x, y) & =h_{1}\left(y_{1}\right)+\ldots+h_{r}\left(y_{r}\right) & & \text { if } y \in D, \\
& =+\infty & & \text { if } y \notin D .
\end{aligned}
$$

For each $y \in D, h_{i}\left(y_{i}\right)<+\infty$ for $i=1, \ldots, r$, and hence the convexity of the $h_{i}$ 's can be used to show directly that the restriction of $\bar{K}(x, \cdot)$ to $D$ is convex and never $+\infty$. Since $D$ is a convex set, this means that $\bar{K}(x, \cdot)$ itself is convex with effective domain $D$. If $x \notin C$, then $x_{j} \notin C_{j}$ for some $j$, so that by (34.3) $h_{j}$ is constantly $-\infty$ on ri $D_{j}$ and hence $\bar{K}(x, \cdot)$ is constantly $-\infty$ on ri $D$. Suppose $x \in C$. Then by (34.3) each $h_{i}$ is proper convex with $D_{i} \subset \operatorname{dom} h_{i} \subset \operatorname{cl} D_{i}$, and moreover $h_{i}$ is closed with $D_{i}=\operatorname{dom} h_{i}$ when actually $x_{i} \in$ ri $C_{i}$. From these facts and (i) and (ii) of Lemma 2 it follows that $\bar{K}(x, \cdot)$ is proper convex, and moreover $\bar{K}(x, \cdot)$ is closed when actually $x \in$ ri $C$. This completes the verification that $\bar{K}$ is concave-closed and satisfies the six conditions of (34.3).

Hence $\bar{K}$ is closed and proper with effective domain $C \times D$ and moreover $\bar{K}$ is concave-closed. Similarly, the function $\underline{K}$ defined in (ii) is convex-closed, closed proper concave-convex with the same effective domain $C \times D$. By (34.4) it follows trivially that $\underline{K}$ and $\bar{K}$ have the same kernel and hence belong to the same equivalence class, call it $[K]$. By (34.2) applied to $\left[K_{i}\right]$ we have $\underline{K}_{i} \leq \tilde{K}_{i} \leq \bar{K}_{i}$ for each $\tilde{K}_{i} \in\left[K_{i}\right]$. Hence by $(34.2)$ applied to $[K]$, it follows that $\left[\left(K_{1}, \ldots, K_{r}\right)\right]$ is 
well-defined and coincides with $[K]$. This completes the proof of (i) and (ii).

Next we prove (iii). By (i) and (37.4), dom $\partial K \subset C \times D$. So suppose $(x, y)$ $\in C \times D$. By (37.4.1) we have

$$
\partial K(x, y)=\partial_{1} \bar{K}(x, y) \times \partial_{2} \underline{K}(x, y)=\partial \bar{K}(\cdot, y)(x) \times \partial \underline{K}(x, \cdot)(y) .
$$

But (iii) of Lemma 2 and (ii) of the theorem imply that

$$
\begin{aligned}
\partial \underline{K}(x, \cdot)(y) & =\partial \underline{K}_{1}\left(x_{1}, \cdot\right)\left(y_{1}\right) \times \ldots \times \partial \underline{K}_{r}\left(x_{r}, \cdot\right)\left(y_{r}\right) \\
& =\partial_{1} \underline{K}_{1}\left(x_{1}, y_{1}\right) \times \ldots \times \partial_{1} \underline{K}_{r}\left(x_{r}, y_{r}\right),
\end{aligned}
$$

where (37.4.1) allows us to replace each $\underline{K}_{i}$ by $K_{i}$. This establishes (iii) for the case $j=1$, and the case $j=2$ is similar.

Finally, we prove (iv) by induction. Observe first of all that (i) and (ii) imply that when $r>2$,

$$
\left[\left(K_{1}, \ldots, K_{r}\right)\right]=\left[\left(H, K_{r}\right)\right], \quad \text { where }[H]=\left[\left(K_{1}, \ldots, K_{r-1}\right)\right] .
$$

Suppose we have already proved (iv) for the case when $r=2$, and let $r>2$. Then by the observation above, the equivalence class conjugate to $\left[\left(K_{1}, \ldots, K_{r}\right)\right]$ is $\left[\left(H, K_{r}\right)^{*}\right]$, which equals $\left[\left(H^{*}, K_{r}^{*}\right)\right]$ by the case $r=2$. But $\left[H^{*}\right]=\left[\left(K_{1}^{*}, \ldots\right.\right.$, $\left.\left.K_{r-1}^{*}\right)\right]$ by the inductive hypothesis, and (since the equivalence classes $\left[K_{1}^{*}\right], \ldots$, $\left[K_{r}^{*}\right]$ satisfy the hypotheses of the theorem) the observation above implies $\left[\left(H^{*}, K_{r}^{*}\right)\right]=\left[\left(K_{1}^{*}, \ldots, K_{r}^{*}\right)\right]$. This completes the inductive step, and so the proof by induction will be complete once we establish the initial step, which is (iv) for the case $r=2$.

Write $[K]=\left[\left(K_{1}, K_{2}\right)\right]$ and $\operatorname{dom} K_{i}^{*}=C_{i}^{*} \times D_{i}^{*}$. Then by (36.3) and (36.1),

$$
\begin{aligned}
\underline{K}^{*}\left(x^{*}, y^{*}\right) & =\sup _{y \in D} \inf _{x \in C}\left\{\Sigma\left\langle x_{i}, x_{i}^{*}\right\rangle+\left\langle y_{i}, y_{i}^{*}\right\rangle-K_{i}\left(x_{i}, y_{i}\right)\right\} \\
& \leq \sup _{y_{2} \in D_{2}} \inf _{x_{2} \in C_{2}}\left\{\left\langle x_{2}, x_{2}^{*}\right\rangle+\left\langle y_{2}, y_{2}^{*}\right\rangle-K_{2}\left(x_{2}, y_{2}\right)+\underline{K}_{1}^{*}\left(x_{1}^{*}, y_{1}^{*}\right)\right\} \\
& = \begin{cases}\sum \underline{K}_{i}^{*}\left(x_{i}^{*}, y_{i}^{*}\right) & \text { if } x_{1}^{*} \in C_{1}^{*} \text { and } y_{1}^{*} \in \operatorname{dom} \underline{K}_{1}^{*}\left(x_{1}^{*}, \cdot\right), \\
+\infty & \text { if } x_{1}^{*} \in C_{1}^{*} \text { and } y_{1}^{*} \notin \operatorname{dom} \underline{K}_{1}^{*}\left(x_{1}^{*}, \cdot\right), \\
-\infty & \text { if } x_{1}^{*} \notin C_{1}^{*} .\end{cases}
\end{aligned}
$$

Moreover, in the event that $x_{1}^{*} \in C_{1}^{*}$ and $y_{1}^{*} \in \operatorname{dom} \underline{K}_{1}^{*}\left(x_{1}^{*}, \cdot\right)$ we have

$$
\begin{aligned}
\sum \underline{K}_{i}^{*}\left(x_{i}^{*}, y_{i}^{*}\right) & =\sum \underline{K}_{i}^{*}\left(x_{i}^{*}, y_{i}^{*}\right) \in R & & \text { if } x_{2}^{*} \in C_{2}^{*} \text { and } y_{2}^{*} \in \operatorname{dom} \underline{K}_{2}^{*}\left(x_{2}^{*}, \cdot\right), \\
& =+\infty & & \text { if } x_{2}^{*} \in C_{2}^{*} \text { and } y_{2}^{*} \notin \operatorname{dom} \underline{K}_{2}^{*}\left(x_{2}^{*}, \cdot\right), \\
& =-\infty & & \text { if } x_{2}^{*} \notin C_{2}^{*} .
\end{aligned}
$$


Also, $D_{i}^{*} \subset \operatorname{dom} \underline{K}_{i}^{*}\left(x_{i}^{*}, \cdot\right)$ for each $x_{i}^{*}$. With $C^{*}=C_{1}^{*} \times C_{2}^{*}$ and $D^{*}=D_{1}^{*}$ $\times D_{2}^{*}$, these facts imply that $\operatorname{dom}_{1} \underline{K}^{*} \subset C^{*}, D^{*} \subset \operatorname{dom}_{2} \underline{K}^{*}$, and

$$
\underline{K}^{*}\left(x^{*}, y^{*}\right) \leq \sum \underline{K}_{i}^{*}\left(x_{i}^{*}, y_{i}^{*}\right) \quad \text { whenever } x^{*} \in C^{*} \text { or } y^{*} \in D^{*} .
$$

Parallel reasoning starting from $\bar{K}^{*}\left(x^{*}, y^{*}\right)$ yields that $C^{*} \subset \operatorname{dom}_{1} \bar{K}^{*}, \operatorname{dom}_{2} \bar{K}^{*}$ $\subset D^{*}$, and

$$
\sum \bar{K}_{i}^{*}\left(x_{i}^{*}, y_{i}^{*}\right) \leq \bar{K}\left(x^{*}, y^{*}\right) \quad \text { whenever } x^{*} \in C^{*} \text { or } y^{*} \in D^{*}
$$

Therefore $\operatorname{dom} K^{*}=C^{*} \times D^{*}$, and for any $\tilde{K}_{i}^{*} \in\left[K_{i}^{*}\right]$,

$$
\underline{K}^{*}\left(x^{*}, y^{*}\right) \leq \sum \tilde{K}_{i}^{*}\left(x_{i}^{*}, y_{i}^{*}\right) \leq \bar{K}^{*}\left(x^{*}, y^{*}\right)
$$

whenever $x^{*} \in C^{*}$ or $y^{*} \in D^{*}$. These facts, together with (34.2) applied to $\left[K^{*}\right]$, imply that $\left[K^{*}\right]$ and $\left[\left(K_{1}^{*}, K_{2}^{*}\right)\right]$ have the same kernel. Since these two equivalence classes are each closed and proper, (34.4) then implies that they are the same. This proves (iv) and completes the proof of Theorem 7.

Proof of Theorem 1. Let $A$ be the product linear transformation

$$
A(x, y)=(x, \ldots, x, y, \ldots, y)
$$

mapping $R^{m} \times R^{n}$ into $R^{p} \times R^{q}$, where $p=r m$ and $q=r n$, and let $[K]$ be $\left[\left(K_{1}, \ldots, K_{r}\right)\right]$. Theorem 1 then follows from Theorem 7 and Theorem 4 applied to this $[K]$ and this $A$.

Joint proof of Theorems 2 and 3. Let $A$ be the product linear transformation

$$
A\left(x_{1}, \ldots, x_{r}, y_{1}, \ldots, y_{r}\right)=\left(x_{1}+\ldots+x_{r}, y_{1}+\ldots+y_{r}\right)
$$

mapping $R^{p} \times R^{q}$ into $R^{m} \times R^{n}$, where $p=r m$ and $q=r n$, and let $[K]$ be $\left[\left(K_{1}, \ldots, K_{r}\right)\right]$. Theorems 2 and 3 then follow from Theorems 5, 6 and 7 applied to this $[K]$ and this $A$.

\section{REFERENCES}

1. J.-P. Gossez, On the subdifferentialof a saddle function, J. Functional Analysis 11 (1972), 220-230.

2. V. N. Lebedev and N. T. Tynjanskiï, Duality theory for concave-convex games, Dokl. Akad. Nauk SSSR 174 (1967), 1264-1267 = Soviet Math. Dokl. 8 (1967), 752-756. MR 35 \#5229.

3. L. McLinden, Minimax problems, saddle functions and duality, Thesis, University of Washington, 1971. (Revised and expanded as Technical Summary Report \# 1190, Mathematics Research Center, University of Wisconsin, Madison, Wis., 1971.)

4._, An extension of Fenchel's Duality Theorem to saddle functions and dual minimax problems, Pacific J. Math. (to appear).

5. J.-J. Moreau, Théorèmes ' inf-sup’, C. R. Acad. Sci. Paris 258 (1964), 2720-2722. MR 28 \# 4336.

6. R. T. Rockafellar, Minimax theorems and conjugate saddle-functions, Math. Scand. 14 (1964), 151-173. MR 30 \#5223. 
7. R. T. Rockafellar, Duality and stability in extremum problems involving convex functions, Pacific J. Math. 21 (1967), 167-187. MR 35 \#2636.

8.-, A general correspondence between dual minimax problems and convex programs, Pacific $\mathrm{J}$. Math. 25 (1968), 597-611. MR 37 \#6110.

9._, Monotone operators associated with saddle-functions and minimax problems, Proc. Sympos. Pure Math., vol. 18, part 1, Amer. Math. Soc., Providence, R.I., 1970, pp. 241-250.

10.— Convex Analysis, Princeton Math. Series, no. 28, Princeton Univ. Press, Princeton, N.J., 1970. MR 43 \#445.

11.—, Saddle-points and convex analysis, Differential Games and Related Topics, American Elsevier, New York, 1971, pp. 109-127.

12. N. T. Tynjanskiī, Conjugate concave-convex functions in linear topological spaces and their saddlepoints, Mat. Sb. 78 (120) (1969), 512-541 = Math. USSR Sb. 7 (1969), 503-531. MR 39 \#3823.

Mathematics Research Center, University of Wisconsin, Madison, Wisconsin 53706 